\title{
Unique Renaming of Java Using Source Transformation*
}

\author{
Xinping Guo \\ James R. Cordy \\ Thomas R. Dean \\ School of Computing, Queen's University \\ Kingston, Ontario, Canada K7L 3N6 \\ \{guo,cordy,dean\}@cs.queensu.ca
}

\begin{abstract}
This paper presents a method for unique renaming declarations and references in Java programs using source transformation to XML markup. Each entity declaration and reference in the Java program is assigned a globally unique identifier (UID) based on its declaration scope and file. The UID serves as a key by which the entity's original declaration and all references can be found, and more importantly, by which information about the entity can be stored or retrieved from the design database. The resulting uniquely renamed source code makes it convenient and efficient to do further business logic and technical analysis that crosses the boundary between source code and the design database.

UIDs are attached to entity references in the source code using XML markup, so that both the UID and the original source text of the declaration or reference are available in the renamed source program. While it is possible to generate unique names in an ad hoc manner, we show how to generate them using a combination of source transformations and design database inferences. This ensures that the notion of UID is consistent and well defined.
\end{abstract}

\section{Introduction}

A common technique in program comprehension is the extraction of a database of design facts from source code. These facts describe the properties of entities in the software system and relations between them. Examples are structural relationships such as methods declared within classes and semantic relationships such as the call relation between methods, or the use relation between methods and variables. The extraction of this database of design facts from source code is known as design recovery [35].

The recovered facts are at a lower level than in a conventional forward design model since developer intention is not explicitly present in the code. Much of the current research in design recovery focusses on analyzing the recovered design database to deduce higher level design information about the system. This analysis involves complex queries such as indirect dependencies, impact analysis, reachability and type analysis.

\footnotetext{
* This research was supported in part by the Natural Sciences and Engineering Research Council of Canada (NSERC).
}

For example, variables representing calendar dates can be identified using a combination of analysis of names, record structure and variable use in comparisons and assignments [9].

While the design database is useful for many tasks that produce summary style reports such as graph visualization and structural analysis, relating the results of the analysis back to the original source code can be difficult. One might want to use the results of the analysis performed on the design database to guide a transformation of the source, or to report analysis results in the context of the source code. While including the abstract syntax tree (AST) in the design database [32, 33] and using lexical coordinates can tie results back to static source [11], this method fares less well when used in the context of source transformation and automated reprogramming tasks.

Unique naming [9] uses a different approach. Unique naming provides a uniform position-independent means of associating the declaring and referencing instances of an entity in the source code with the corresponding entity in the design database. Using unique naming, all instances referring to an entity in both the source and the database use the same unique name, which serves as a kind of key linking the one to the other.

This representation has two quite distinct advantages - first, because analysis artifacts are represented with both the source and design representations, the database need not be constrained to carry source information, and the source need not be restructured to match the design representation, making it easier to analyze and transform each. Secondly, because source and design are implicitly linked to one another by the unique names of the entities, analysis and transformation of source and analysis and transformation of design can each be carried out independently without losing the connection to the other. Tasks more appropriate to source can be carried out on source, and tasks more appropriate to design can be carried out on the design database. In either case, the changed result of tasks carried out on one are automatically attached to the other by the unique names of the entities involved.

In order to be effective, unique names must provide entity identifiers that are globally unique across entire systems, while at the same time easy to derive locally so that source files can be uniquely named on a file by file basis. One way to do this is to derive the unique identifier (UID) of each entity from the source file and scope context of its defining declaration, in the style of internet URLs. 
By encoding the UIDs in source declarations and references using markup rather than replacement, we preserve the original source text of each reference and declaration in the uniquely named source while attaching each to the design database. Unique naming using markup has been used for COBOL, RPG and PL/I systems in LS/2000 [9] and has been extended to complex analysis of maintenance hotspots in these languages using HSML [31].

While unique naming has worked well for analysis and transformation of these older procedural languages, legacy systems in object-oriented languages such as Java [27] are an increasing concern. In this paper we extend the scope-based unique naming of these older languages to handle the more complex scope and inheritance rules of modern object-oriented languages, and show how object-oriented linking can be reflected directly into source using unique name resolution. We introduce a method for resolution of unique names in the presence of class instances and inheritance, and extend the notion to handle shared object-oriented class libraries.

We use XML [1] tags as the markup notation to add unique naming tags to Java source. XML is already widely used in reverse engineering systems to represent both syntactic analysis information and software designs [2,3,4,5,6,14]. XML tags provide a flexible way to represent many kinds of information, such as syntax and semantic analysis information, design graphs, and source elisions, all of which can be built on our unique renaming.

\section{Unique Identifiers (UIDs)}

Java program comprehension and Java design recovery are currently popular research topics. These activities become more challenging when working with Java source artifacts because of the use of name spaces. For example, it is possible that all of the following names in a Java program could be the same:

- class, constructor and field names in a class

- two class names in different packages

- overloaded method names

- local variables in different methods

- parameters / local variables and class / instance variables

One way to distinguish between these entities is by using scope rules to give each declared name a UID [9]. Sample Java source code and generated UIDs are shown in Figure 1. We use this sample throughout the paper to demonstrate the process of generating and manipulating UIDs.

In our approach, the UID of an entity is based on the original name of the entity, along with information encoding the entity's scope and location in the code, in the form:

\section{"entity_name enclosing_class_and_interface_names package_name file_name"}

Using this approach, in the example of Figure 1 we can see:

- The class and local variables named $x$ are assigned the different UIDs, "x Ex bar foo Ex.java" and "x main Ex bar foo Ex.java",

- The local variables named $y$ in the two methods are assigned the different UIDs, "y $x$ Ex bar foo Ex.java" and "y main Ex bar foo Ex.java".

Two classes in different packages with same name would also have different UIDs because the package names are included in the UIDs, and so on. However, in the example we

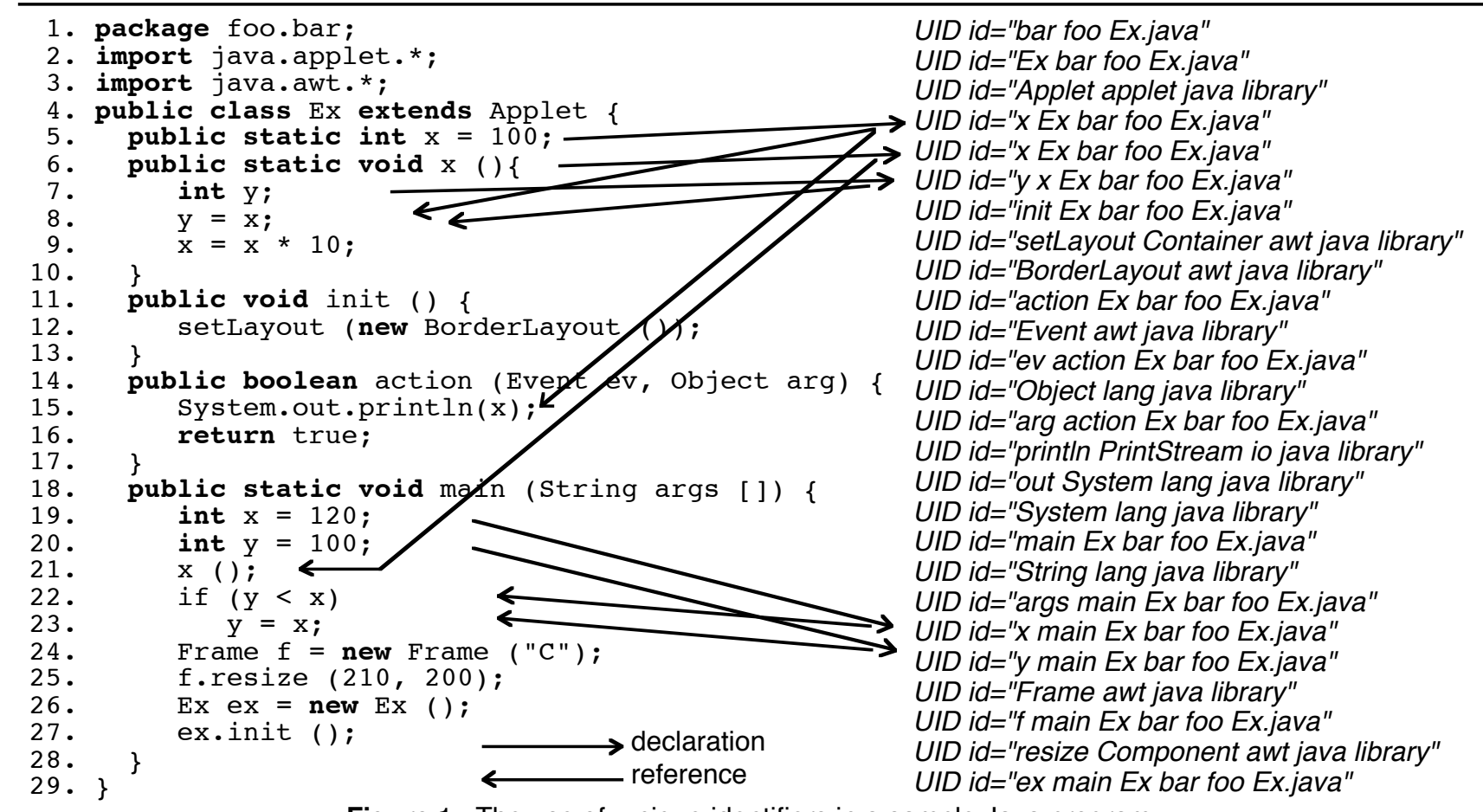

Figure 1. The use of unique identifiers in a sample Java program. 
see that both the field and the method $x$ have been assigned the same UID " $x$ Ex bar foo Ex.java". The reason is that it is not necessary to distinguish these two, because in both source code and the design database it is unambiguous which is meant in every context. In source code, field references and method references are easily distinguished because method declaration and invocation always includes parentheses with zero or more arguments. In the design database, field and method entities are contextually distinguished by the database schema. This was a conscious decision in our model. If necessary, method and field UIDs can be distinguished simply by adding the word "method" or "field" in the UID strings as appropriate.

Overloaded methods are assigned the same UIDs in this schema as well, because this makes working with our reference design model simpler. If the distinguishability is important, we can modify our model to recognize the signature match.

\section{The Java Unique Renaming Process}

Our unique renaming process involves assigning UIDs to all declared entities in Java source code and libraries. UIDs are assigned based on declaration context in the source code. References in the code to external and library entities must also be annotated.

There are eight steps in Java unique renaming process, described in detail in the eight following sections. The eight steps are as follows:

1. Uniquely rename declarations in each class file.

2. Uniquely rename internal direct references to declarations in each class file.

3. Uniquely rename the Java library interfaces (once for all programs).

4. Uniquely rename external direct references to library and other class files.

5. Uniquely rename qualified references in each class file.

6. Extract a simple data design model from the renamed library and all program class files.

7. Analyze the data model to link all external reference unique names to their external target library and class file entities.

8. Implement the links in each class file to replace external reference unique names with the unique names they are linked to.

When the process is complete, every entity declaration and reference in every class file in the program will be globally uniquely renamed to refer directly to the UID of the referenced entity.

\subsection{Step 1: Rename Declarations}

The goal of this first step is to assign each declared name in each class file a UID in the source code. The entities to be annotated include package, class, interface, variable, constructor, and method. UIDs are created layer by layer from the outside in.
Adding file and package names is straightforward; however, adding other information is tricky, especially for inner classes. The use of recursive transformation rules is a good solution for handling these cases. We begin by annotating all declarations with XML tags containing partial UIDs, of the form:

$$
<U I D \text { id= "file_name" }>\text { declared_name }</ U I D>
$$

We then incrementally add each level of scope information to the UIDs in the XML tags one level at a time by processing each scope of the program from the outside in. The final result UID encodes the file name, declared name and the names of all enclosing scopes in inside out order:

$$
\begin{aligned}
& <U I D \text { id= "declared_name inner_scope_name ... } \\
& \text { outer_scope_name package_name file_name" }> \\
& \text { declared_name }</ U I D>
\end{aligned}
$$

Figure 2 shows the annotated source code from this first step for the sample program in Figure 1. When we are done, the UID for each declared entity represents all of its scope information. For example, the UID " $y x$ Ex bar foo Ex.java" means that local variable $y$ is located in method $x$, method $x$ is in class $E x$, class $E x$ is in package foo.bar and the source class file name is Ex.java. After this step UIDs have been assigned to all declared names, but references have still to be renamed (Figure 2).

All declared names inside class Ex contain "... Ex bar foo Ex.java" in their UIDs. All declared names nested in a method contain the method name as well. For example, in the main() method, all UIDs of declarations end with "... main Ex bar foo Ex.java". This property makes our UIDs a rich source of scoping information which can be exploited to optimize source analysis and transformation tasks by avoiding reference to the design database to look up structural information such as containment relationships.

\subsection{Step 2: Rename Internal Direct References}

Once all declarations have been annotated with their unique names as shown in Figure 2, we must annotate direct references to these declared names in each class file. Direct references are leftmost unqualified names of reference expressions, for example, Applet, $y$, and $f$ in f.resize(). We will uniquely rename the qualifications in a later stage of the process.

Most references are internal - references to declared names in the class file itself (e.g. variables $y$ and $f$ ), but others are external references to entities in the library (e.g. Applet and Frame) or other class files. Binding references to declarations must of course follow the scope rules of the language, so the correct UID for any reference is the UID of the declaration of the entity to which the reference refers. For example, in Figure 1 , the $x$ in the statement $x=x^{*} 10$ is a reference to the variable $x$ declared in the statement public static int $x=100$, so the UID for the reference is " $x$ Ex bar foo Ex.java".

The steps in renaming of references mirror the steps in the scope rules of Java. We begin in this section with the simplest case, resolving direct references to already uniquely named declarations and annotating each with the UID of the declaration. Unique naming of references to libraries and external packages is handled in the following sections. 


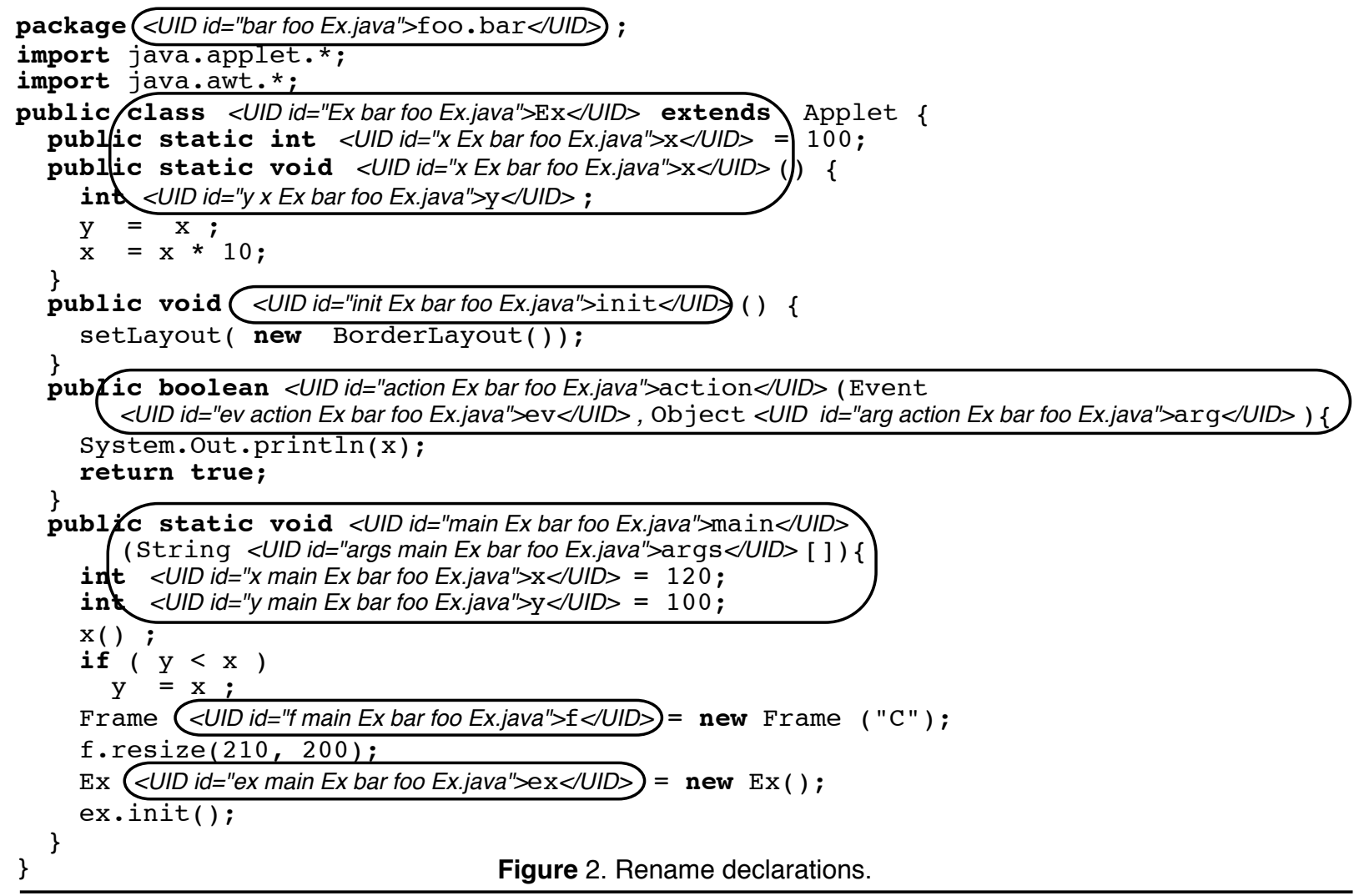

We tag each internal direct reference with the UID of the declared entity it refers to, if any. If none is found, the reference is assumed to be external and is handled in the step 4 . When this step is complete, all declarations and their internal direct references in each class file have been annotated with UIDs as shown for our sample code example in Figure 3.

While direct references to $x$, class $E x$ and local variables $x, y$, $f$ and $e x$ have been uniquely renamed in the source as shown in Figure 3, there still remain several unresolved references. These are of two kinds. First, there are references to entities contained in Java libraries and other class files such as classes (Applet, System, Frame) and inherited methods (setLayout()). Second, there are qualifiers in compound references, such as method and field names. Examples are resize() in f.resize() and init() in ex.init(). We continue by addressing the first issue, references to external class files and Java libraries.

\subsection{Step 3: Rename Java Library Interfaces}

In order to accurately uniquely rename external references to the library in Java source code, we must first address the question of uniquely renaming the Java library files themselves. We need only do library unique renaming once - we can store the results and use the same unique names to resolve external Java library references in all Java programs.

A difficulty with this approach is the fact that we do not have the sources for all of the Java libraries. As it turns out, this is not a serious problem - we can use the javap command to disassemble the Java class files of the library to source interfaces and rename those instead. The output of javap is an interface source file similar to the original library source code but without package name and implementation details. We simply use the declaration and reference unique renaming transformations described in Sections 3.1 and 3.2 to implement unique renaming of the library. In place of the source class file name, the special file name "library" is used in all library UIDs to ensure consistency among library files.

\subsection{Step 4: Rename External Direct References}

Once we have the uniquely renamed libraries, we are ready to uniquely rename external references in any Java program. Once again, at this stage we only rename direct references (leftmost unqualified names in reference expressions) in each class file. Qualifications will be uniquely renamed in step 5 . The result of these two steps on our sample program is shown in Figure 4.

We begin by uniquely renaming all references to types (class types and interface types) declared in the library and other class files. We do this by resolving the names used in all still remaining unrenamed direct references using the declarations from the uniquely renamed libraries created in step 3 and other annotated Java files created in step 2.

In the sample program of Figure 4, references to Applet, BorderLayout, Event, Object, System, String and Frame have all been uniquely renamed as library references. For example, the UID of Applet was found to be "Applet applet java library", denoting a reference to the Applet class of the Java library. 


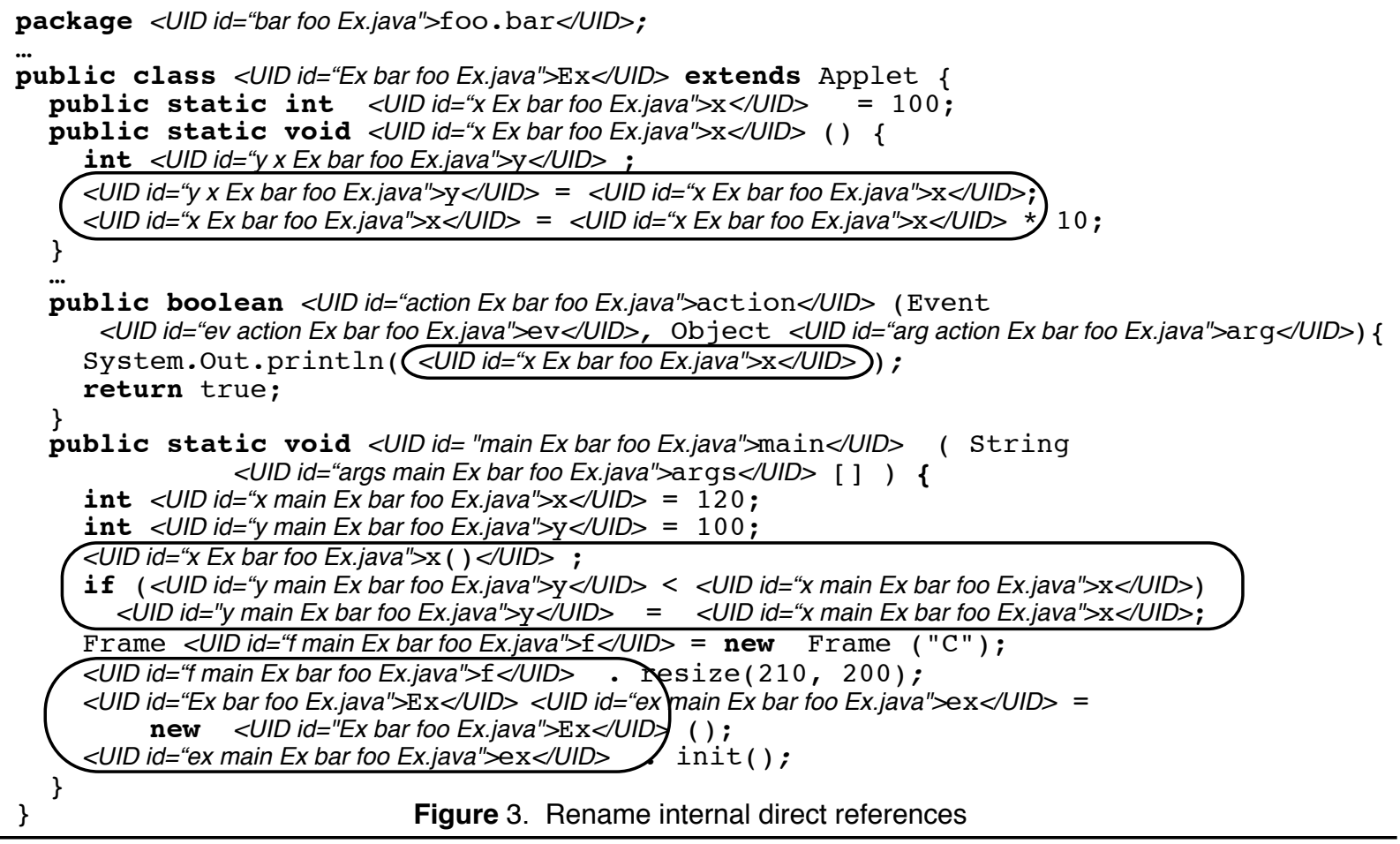

Once we have resolved all library references, the only remaining unresolved direct references must be to external user symbols such as inherited members of other class files. To complete the unique renaming of references, we assign each of these references a temporary UID as if they were declared in current class, for example, the reference to the inherited member setLayout() of our sample program is approximated as "setLayout Ex bar foo Ex.java". Section 3.7 describes our strategy for the linking of these temporary UIDs to the actual external entities they refer to in other class files.

\subsection{Step 5: Rename Qualified References}

Once unique names have been assigned to all external references, there remain no unresolved direct references in the program. At this point we are ready to tackle refinement of UIDs for qualified references, e.g. System.out.println(), f.resize() and ex.init(). Previous steps have already uniquely renamed the direct (or base) reference of every qualified name. For example,

$$
<\text { UID id= "System lang java library" }>\text { System }</ U I D>
$$$$
\text { .out.println ( ) }
$$

In this step we expand these base references to create UIDs for the entire qualified references level by level. This is actually a very simple process. Since Java does not allow partial qualification, the UID for a reference $x . y$ where $x$ has UID " $x$ blat bar foo prog.java" is always simply " $y x$ blat bar foo prog.java", that is, $y$ followed by the UID for $x$.

By applying this algorithm at every level, we get fully renamed qualified names. For example, the uniquely named qualified expression System.out.println() shown above becomes:

$$
\begin{gathered}
<\text { UID id= "println out System lang java library" }> \\
<U I D \text { id= "out System lang java library" }> \\
<\text { UID id }=\text { "System lang java library" }> \\
\text { System }</ \text { UID }>\text {.out }</ \text { UID }>\text {.print } \ln ()</ \text { ID }>
\end{gathered}
$$

The result of the resolution of qualified names for our sample program is shown in Figure 4.

While this simple qualification naming algorithm works correctly for most internal qualified references, at this stage UIDs for qualified references are really an approximation. Firstly, qualified object references have been renamed as if the fields and methods were members of the object, when in fact they should be renamed to refer to the corresponding class member declaration. Secondly, external references are an approximation because we have not yet taken into account inheritances that may be present in the external class hierarchies.

For example, the UID "println out System lang java library" should really refer to "println PrintStream io java library". In the next two sections, we address both these issues by implementing a link analysis of the entire renamed data model of all program class files and the library together.

\subsection{Step 6: Extract the Data Design Model from Renamed Source Files}

At this point in the unique renaming process we have assigned unique names to every declaration and reference in all class files of a Java program. However, references to qualified and inherited members have been represented by internal approximate UIDs. The problem now is to link these approximate local UIDs to the entities to which they refer. In order to do this, we use a static data design model to imitate the actions of a Java run-time linker. 


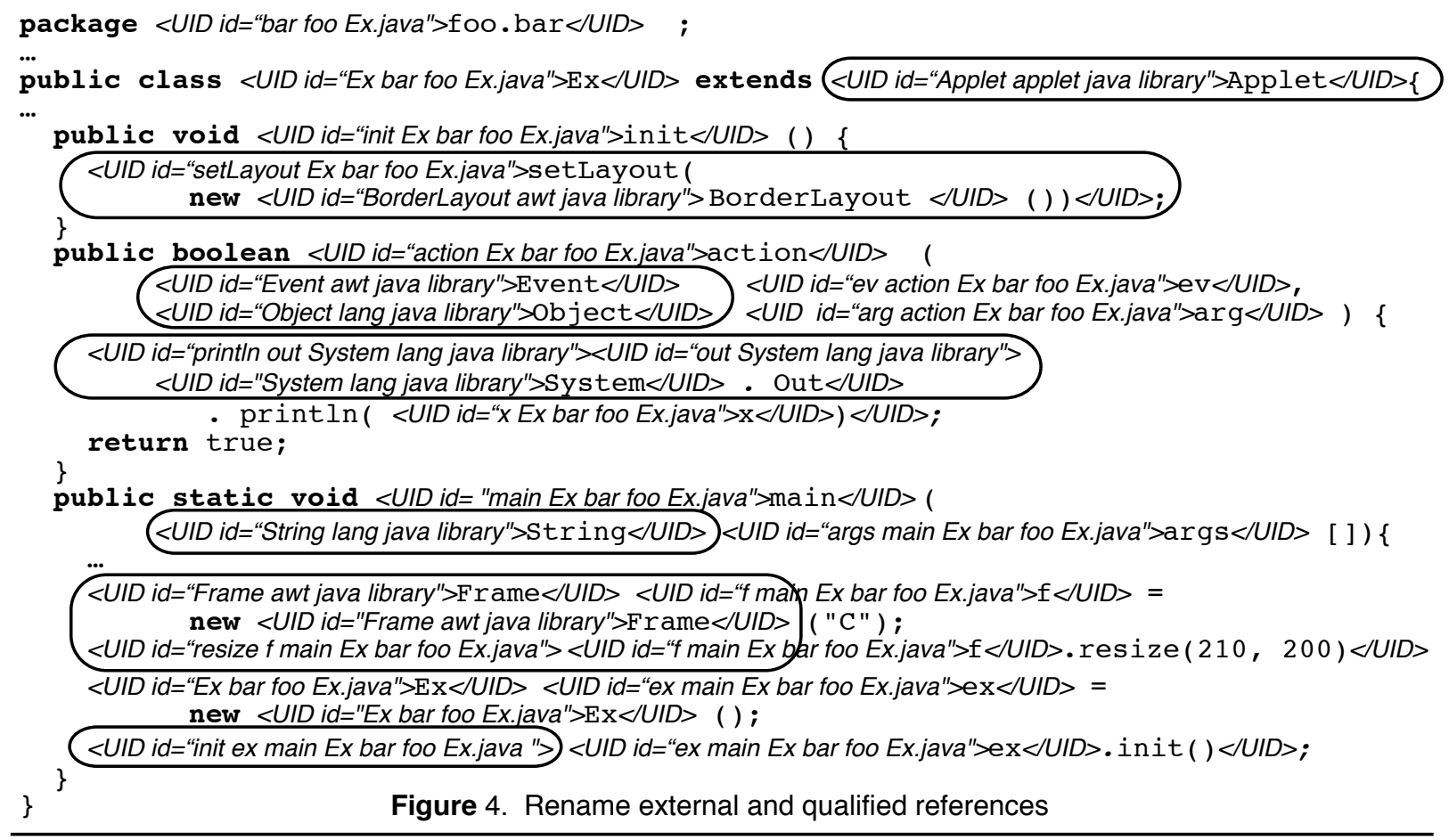

We begin by extracting a database of data design facts from each of the uniquely renamed source files in the program. This database can be useful in many design analysis tasks, but in particular, we can use it to resolve the actual entity targets of our approximate external UIDs in section 3.7.

We use the design recovery technique described by Schneider et al. [29] to infer and gather data design facts from our uniquely renamed source and library files. TXL rules are used to search for patterns in the source and annotate the source with design facts [8].

In the following paragraphs, we describe the facts inferred in our data design recovery for linking. Figures 5 and 6 show examples of the data design facts extracted from the library and class files of our sample program.

Entity Facts. We extract entity facts from each of the renamed library files and class files. These facts describe the defined packages, classes and interfaces in the files.

Type Structure Facts. The type structure facts describe the relationships between entities and types. These include inheritance, abstraction, members and inner types. We derive the following facts:

- hasFieldType - Represents the types of declared fields.

- hasMethodType - Represents the types of declared methods.

- hasMemberForClass - Represents the member relationship.

- hasInnerType - Represents the inner class relationship .

- hasSuperType - Represents the extends relationship.

- hasImplement-Represents the implements relationship.

Method Facts. Parameter, local variable and type facts are extracted at the level of methods/constructors. These facts encode not only implementation detail but also relationships with other classes and interfaces.

- paramVar \& localVar - Method contains parameter and local variable.

- funRef-The method references another method.

- varRef - The variable references in methods.

- typeRef - Representing class or interface references particularly in class instance creation expressions and casting conversions.

- genRef - The method contains references that need to be generalized.

- varType - The type of the variable.

All unknown entities, which link to external entities or the library, are included in the facts funRef, varRef and genRef. They might be inherited members or they might come from qualified references. They will be resolved to their exact UIDs in the next section. So far, from source code we have derived that each funRef refers to an internal or external method, each varRef refers to an internal or external field, and so on. But genRef facts are ambiguous, meaning that the correct semantics for the reference has yet to be determined. We do not know if the entity refers to a class type, package, field, or method.

\subsection{Step 7: Deriving Link Relationships}

Based on the data design model (i.e. the facts) recovered from the source and library files in Section 3.6 (Figure 5, Figure 6 ), we can derive the final linking relationships between class instance and external entity references. Those external entities are defined in libraries or other source files of the program. Once we have determined these links, we can replace the approximate UIDs generated for these references in Section 3.5 
hasSuperType ("System lang java library", "Object lang java library")

hasMemberForClass ("System lang java library", "out System lang java library")

hasFieldType ("out System lang java library",

"PrintStream io java library")

hasSuperType ("Panel awt java library", "Container awt java library") hasSuperType ("Applet applet java library", "Panel awt java library")

hasMemberForClass ("Applet applet java library", "init Applet applet java library")

hasMethodType ("init Applet applet java library", void)

hasSuperType ("Container awt java library", "Component awt java library")

hasMethodType ("setLayout Container awt java library", void)

hasMemberForClass ("Container awt java library", "setLayout Container awt java library")

hasMethodType ("action Component awt java library", boolean)

hasMemberForClass ("Component awt java library", "action Component awt java library")

Figure 5. A subset of the facts for the Java library.

by the UIDs of their actual target entities. The link relationships are derived by encoding Java linking rules as inference rules using the Tarski relational algebra system Grok [10,24].

Linking Through Inheritance. Some external fields and methods are inherited from super classes. In a current class, an entity can be used as a reference which refers to an entity defined in a super class. For example, setLayout() is an inherited method from java.awt.Container.

Input to our Grok scripts includes all the facts recovered from all program class files and the library. We start by identifying all the temporary references that need to be linked. These references include both the qualified object references and the qualified external references in all of the renamed class files. The result of this analysis is stored in the relationship needlink, which documents all of the approximate UIDs for which we need to resolve a target entity.

We compute the needlink relationship using Grok to find those tuples in funRef, typeRef, varRef and genRef for which there is no directly defined entity in any program class file or library file. Thus, in our sample example, the derived needlinks are:

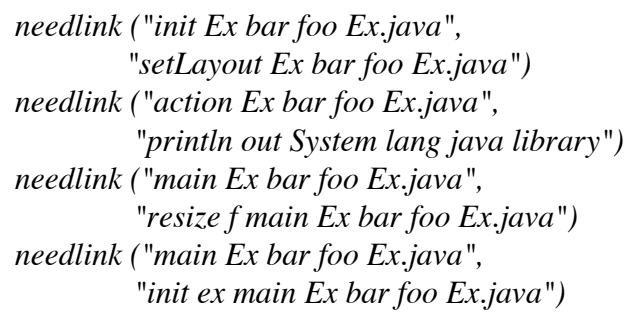

We now have all the information needed in order to actually resolve the links. We use Grok to explore the class hierarchy to resolve references to inherited (super class) methods and fields. We encode the results of this analysis as the superLink relationship. In our example, we obtained:

superLink ("setLayout Ex bar foo Ex.java", "setLayout Container awt java library")

Linking Through Object Instances of Classes. The only remaining unresolved links are qualified object references
hasSuperType ("Ex bar foo Ex.java", "Applet applet java library") hasFieldType ("x Ex bar foo Ex.java", int)

hasMethodType ("init Ex bar foo Ex.java", void)

hasMethodType ("action Ex bar foo Ex.java", boolean)

hasMemberForClass ("Ex bar foo Ex.java", "x Ex bar foo Ex.java")

hasMemberForClass ("Ex bar foo Ex.java", "init Ex bar foo Ex.java")

hasMemberForClass ("Ex bar foo Ex.java", "action Ex bar foo Ex.java")

paramVar ("action Ex bar foo Ex.java", "ev action Ex bar foo Ex.java")

localVar ("main Ex bar foo Ex.java", "x main Ex bar foo Ex.java")

funRef ("init Ex bar foo Ex.java", "setLayout Ex bar foo Ex.java")

funRef ("action Ex bar foo Ex.java", "println out System lang java library"

funRef ("main Ex bar foo Ex.java", "init ex main Ex bar foo Ex.java") genRef ("action Ex bar foo Ex.java", "System lang java library")

genRef ("action Ex bar foo Ex.java", "out System lang java library")

typeRef ("main Ex bar foo Ex.java", "Frame awt java library")

varType ("ev action Ex bar foo Ex.java", "Event awt java library")

varType ("arg action Ex bar foo Ex.java", "Object lang java library")

Figure 6. A subset of the facts for the sample program.

referring to the members of an object's class or super classes. To resolve these references, we use Grok once again to explore the object's class hierarchy. We start with the class of the object itself and look upward until the member it refers to is found. We encode the results of this analysis in the classLink relationship. In the sample program, we derive the links:

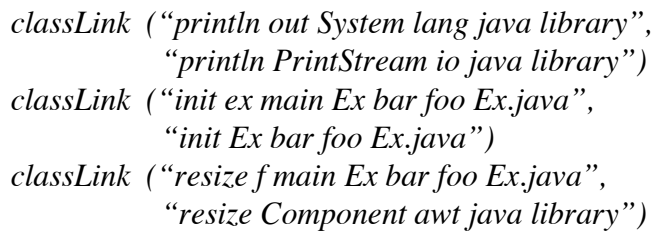

\subsection{Step 8: Push Links into Uniquely Renamed Source}

Based on the links found, the approximate UIDs are replaced in the renamed source. Those approximate UIDs used for object and external qualified names in the uniquely renamed source are replaced by the UIDs of the corresponding actual external entities derived by the linking process. This is done using a final TXL source transformation that uses the inferred link facts of the previous section to replace each linked UID instance in the source with the UID that it is really linked to. In the case of our sample program, we come up with the final uniquely renamed Java source code of Figure 7. The final annotated source code is about five times larger than the original.

\section{Implementation}

Our unique renaming is implemented using a sequence of source transformations written in the TXL language. Data design recovery from the initial renamed source yields a set of base facts used as input to a Grok script to infer links between external references and the appropriate external entities.

A TXL program has two parts: an arbitrary context free grammar (in BNF-like notation) and a set of by-example transformation rules. TXL uses the grammar to automatically parse input files containing source code and then successively applies the transformation rules to the parsed input until the rules fail. The transformed source is then output as annotated code. 


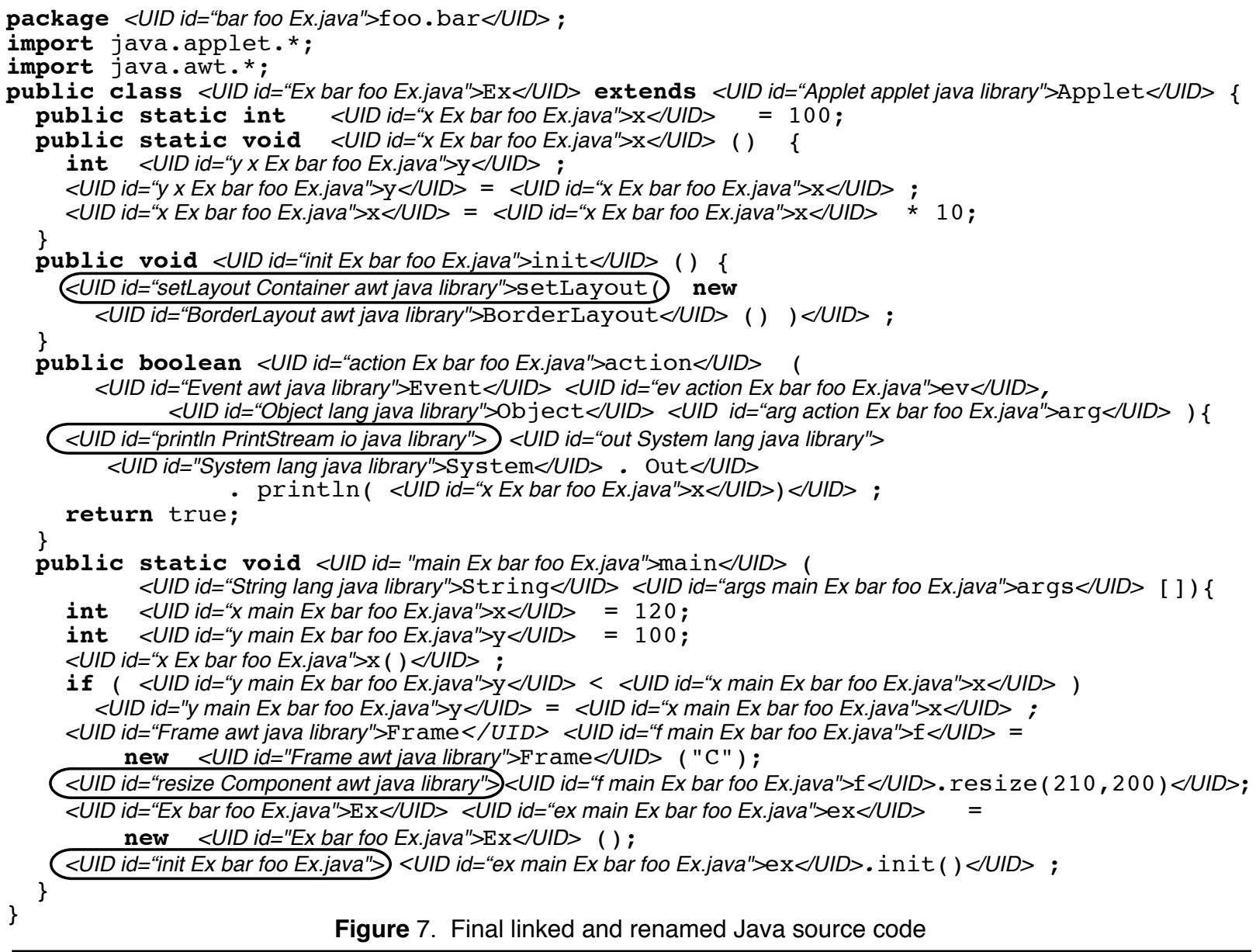

Our TXL Java grammar [23] is based on the Java language specification [13]. We extend the nonterminal definitions of the Java grammar to allow transformation rules to add XML tags around entities. For example, step one (annotate all declarations with the file name) uses the following rule:

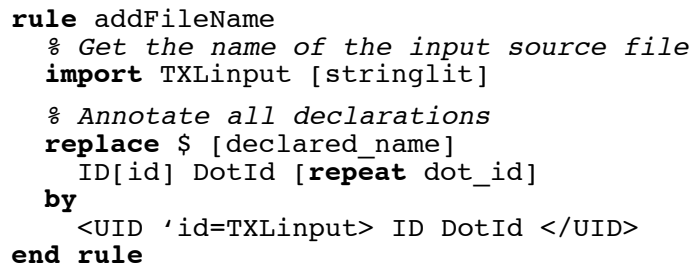

The global variable TXLinput contains that name of the input file and the rule finds each declaration and adds the XML annotation to the name of the declared entity.

\section{Related Work}

The unique renaming paradigm and UID approach on which our work is based was originally designed by Schneider [29] for design recovery and analysis of programs written in the Turing programming language. LS/2000 [9] was a TXL-based process that used similar design recovery techniques to analyze source code for Year 2000 risks. This process guided source transformations that were able to automatically migrate over 99\% of the Year 2000 risks in over three billion lines of production source written in COBOL, PL/I and RPG. The use of UIDs to link between source code and design databases was further explored in the HSML language [31]. The main contribution of this paper to the LS/2000 work is the extension of the unique naming concept to the object oriented language constructs present in Java and not in COBOL, PL/I or RPG. The work in this paper also addresses the more flexible relationships possible between Java source entities that are also not present in the languages supported by LS/2000.

Cox and Clarke [11] developed the Jupiter repository system. Maia is a data model that is encoded using XML-like markup. Tags are used to mark entities such as blocks, declarations, and control flow. Source tokens are numbered sequentially and the markup tokens are assigned fractional token positions based on these source token numbers. Links between tokens are done using attributes that give the source token positions. So a declaration is annotated with markup tokens that give the source token positions of the use of the entity while references tokens are annotated with markup that gives the declaration of the entity. The approach has the flexibility that the tags may be stored in the source code or separately in a design database. The 
disadvantage of using token positions in the references is that the markup is more sensitive to changes in the code.

Middle level models such as the Dagstuhl Middle Model (DMM) [30] encode the source position of entities in the model. In DMM, model objects are associated with source objects using the defines and declares relations. The source objects in the design database have an identity of their own and are linked to the source code by attributes defining the start and end position as line and column numbers. Datrix [32,33], an abstract syntax graph (ASG) approach, also stores the source code locations directly in the model as line and column attributes. Both of the DMM and Datrix approaches share the disadvantage of Maia. One could argue that the ASG based models do not need the link to the source code other than for reporting purposes, since they are source code complete. Any transformations could be done entirely in the design database. However, the fixed schema (i.e. fixed grammar) of the design database limits some techniques that can be used to simplify transformations [34].

There are many papers that explore how to represent source code information for different languages in XML format. Power and Malloy [2] modify the GNU bison parser generator to generate parse trees in XML format for $\mathrm{C}$, Objective $\mathrm{C}, \mathrm{C}++$, Java and FORTRAN. Another program analysis tool, XMLizer [3], also outputs XML format to represent program structure for Java, PL/IX and Pascal. In both cases the XML is used to represent the parse tree, and does not contain any attributes linking the use of an identifier to its declaration. Power and Malloy absorb all of the source text into tags and attributes. XMLizer has the ability to represent partial parse trees. For example, a statement nonterminal may mark text for an entire statement with no parse representation embedded in the statement. When fully parsed, most text is absorbed into XML tags and attributes, although constant and identifiers remain as marked up text. JavaML [14] uses a similar representation as Power and Malloy, but includes a unique identifier in each attribute. This attribute links variables and methods within a file. Methods between files are not attributed.

There are many techniques and tools for source analysis of Java. Sun's JavaCheck [15] can analyze the use of library APIs for compatibility. SHriMP [16, 17, 18], Chava [19], GUPRO $[20,21]$ and the Software Bookshelf [22] are tools that can extract and visualize information from Java programs.

\section{Conclusions and Future Work}

We have described a unique renaming system for Java programs that accurately resolves relationships between program entities in source using unique names (UIDs). Each declared name and reference is annotated with its unique name in the source using XML markup. The UIDs serve as keys uniquely identifying each program entity in both the source and the design database. They form a kind of bridge between the two which allows for independent processing of both source and design without losing the connections between the two. The fully linked uniquely named source representation of the program is suitable for complex program comprehension, analysis, visualization and transformation tasks.
Renamed Java programs localize scope and linking information at the point of reference. Both declarations and references are clearly marked with the UIDs of the entities to which they refer, in order to free further analysis from worrying about name ambiguities. Renamed code can be easily parsed at different levels (light, middle or heavy weight) as either Java source or an XML document. Because unique naming is represented entirely as XML markup of original source text, output of subsequent analysis or transformation tasks can easily include or exclude UIDs in results. Unique renaming can be easily integrated to other reverse engineering tools. For example, very little modification of our data design facts would be input to Rigi [28] or van Emden and Moonen's code smell detection process [12].

The unique renaming described in this paper has thus far been used for only one actual application, a system to assist in Java library version migrations. Using unique renaming of different versions of the AWT library and source programs using it, an accurate analysis of AWT version dependencies and migration path was easily derived. The technique is completely generic and can be used for any other library version migration.

In future work, we may consider adding resolution to distinguish overloaded methods with different UIDs. Also, thus far our method is based entirely on static analysis. For some applications it would be more useful if information from dynamic analysis were added as well. For example, Java reverse engineering projects based on Java byte code [25, 26] could provide more facts to enrich the data model.

We believe that unique renaming is a very basic and important step in Java design recovery and analysis. For example, renamed Java code already has the class dependency information necessary to derive UML or other representations of the program design [25]. We also hope to explore and exploit the properties of uniquely renamed code to make more effective use of existing analysis tools and techniques in the coming years.

\section{Acknowledgments.}

This paper has been significantly improved by the numerous helpful and specific suggestions of the anonymous referees, for which we thank them.

\section{References.}

[1] World Wide Web Consortium. Extensible Markup Language (XML). http://www.w3.org/xml/

[2] J. F. Power and B. A. Malloy. Program annotation in XML: a parse-tree based approach. 9th Working Conference on Reverse Engineering (WCRE 02), pp. 190-198, Oct. 2002.

[3] G. McArthur, J. Mylopoulos, and S. K. K. Ng. An Extensible Tool for Source Code Representation Using XML. 9th Working Conference on Reverse Engineering (WCRE 02), pp.199-208, Oct. 2002.

[4] A. Asencio, S. Cardman, D. Harris, and E. Laderman. Relating Expectations to Automatically Recovered Design Patterns. 9th Working Conference on Reverse Engineering (WCRE 02), pp. 87-96, Oct. 2002. 
[5] C. Riva and Y. Yang. Generation of Architectural Documentation using XML. 9th Working Conference on Reverse Engineering (WCRE 02), pp. 161-169, Oct. 2002.

[6] E. Mamas and K. Kontogiannis. Towards Portable Source Code Representations Using XML. 7th Working Conference on Reverse Engineering (WCRE 00), pp. 172-182, Nov. 2000.

[7] TXL Project, The TXL Programming Language, Version 10.2. http://www.txl.ca/docs/TXL102LangRef.pdf, Apr. 2002.

[8] J.R. Cordy, T.R. Dean, A.J. Malton and K.A. Schneider. Source Transformation in Software Engineering using the TXL Transformation System. Special Issue on Source Code Analysis and Manipulation, Journal of Information and Software Technology 44(13), pp. 827-837, Oct. 2002.

[9] T.R. Dean, J.R. Cordy, K.A. Schneider and A.J. Malton. Experience Using Design Recovery Techniques to Transform Legacy Systems, IEEE International Conference on Software Maintenance (ICSM 2001), pp. 622-631, Nov. 2001.

[10] R.C. Holt. Structural Manipulations of Software Architecture Using Tarski Relational Algebra. 5th Working Conference on Reverse Engineering (WCRE 98), pp. 210-219, Oct. 1998.

[11] A. Cox and C. Clarke. Representing and Accessing Extracted Information. IEEE International Conference on Software Maintenance (ICSM 2001), pp. 12-21, Nov. 2001

[12] E. van Emden and L. Moonen. Java Quality Assurance by Detecting Code Smells. 9th Working Conference on Reverse Engineering (WCRE 02), pp. 97-106, Oct. 2002.

[13] J. Gosling, B. Joy, G. Steele and G. Bracha. Sun Microsystems Inc. The Java Language Specification (2nd edition). Addison Wesley, 2000.

[14] G.J. Badros. JavaML: A Markup Language for Java Source Code. 9th International World Wide Web Conference, pp. 159177, May 2002.

[15] Sun Microsystems Inc. JavaCheck - Platform Compatibility Insurance for your Applications and Applets. http://java.sun.com/products/personaljava/javacheck.html.

[16] M.-A. D. Storey, H. A. Müller and K. Wong. Manipulating and Documenting Software Structures. Series on Software Engineering and Knowledge Engineering, Vol. 7 Software Visualization, pp. 244-263, Nov. 1996.

[17] J. Michaud, M.-A. Storey and H. Muller. Integrating Information Sources for Visualizing Java Programs. IEEE International Conference on Software Maintenance (ICSM 2001), pp. 250-259, Nov. 2001.

[18] University of Victoria. SHriMP Views. http://shrimp.cs.uvic.ca/.

[19] J. Korn. Chava: Reverse Engineering and Tracking of Java Applets. 6th Working Conference on Reverse Engineering (WCRE 99), pp. 314-325, Oct. 1999.

[20] C. Lange, H. M. Sneed and A. Winter. Comparing graphbased program comprehension tools to relational database-based tools. 9th International Workshop on Program Comprehension (IWPC 01), pages 209-218, May 2001.
[21] GUPRO - Generic Understanding of PROgrams. http://www.uni-koblenz.de/ ist/gupro.en.html.

[22] J. Finnigan, R.C. Holt, I. Kalas, S. Kerr, K. Kontogiannis, H.A. Müller, J. Mylopoulos, S.G. Perelgut, M. Stanley, K. Wong. The Software Bookshelf. IBM Systems Journal 36(4), pp. 564-593, Nov. 1997.

[23] TXL Project, The TXL Grammar Collection. http://www.txl.ca/nresources.html.

[24] R.C. Holt. Introduction to the Grok Language. http://plg.uwaterloo.ca/ holt/papers/grok-intro.html.

[25] L. A. Barowski and J. H. Cross II. Extraction and Use of Class Dependency Information for Java. 9th Working Conference on Reverse Engineering (WCRE 02), pp. 309-315, Oct. 2002.

[26] D. Rayside, S. Kerr and K. Kontogiannis. Change and adaptive maintenance detection in Java software systems. 5th Working Conference on Reverse Engineering (WCRE 98), pp. 10-19, Oct. 1998.

[27] Sun Microsystems Inc. The Java Language: An Overview. http://java.sun.com/docs/overviews/java/java-overview-1.html.

[28] M.-A. D. Storey, K. Wong, and H. A. Mller. Rigi: A visualization environment for reverse engineering. 19th International Conference on Software Engineering (ICSE '97), pp. 606--607, May 1997.

[29] J.R. Cordy and K.A. Schneider. Architectural Design Recovery Using Source Transformation, 7th International Workshop on Computer Aided Software Engineering (CASE'95) Workshop on Software Architecture, Toronto, July 1995.

[30] T.C. Lethbridge et al. The Dagstuhl Middle Model version 0.005, http://scgwiki.iam.unibe.ch:8080/Exchange/uploads/2/ DMMDescriptionV0005.pdf

[31] J.R. Cordy, K.A. Schneider, T.R. Dean and A.J. Malton. HSML: Design Directed Source Code Hot Spots. 9th International Workshop on Program Comprehension ( IWPC 2001), pp. 145-154, May 2001.

[32] Bell Canada. DATRIX ${ }^{\mathrm{TM}}$ Abstract Semantic Graph Refernece Manual Version 1.4. Bell Canada, Inc., Montreal, May 2000.

[33] T.R. Dean, A.J. Malton and R.C. Holt. Union Schemas as a Basis for a C++ Extractor. 8th Working Conference on Reverse Engineering (WCRE 2001), pp. 59-67, Oct. 2001.

[34] T.R. Dean, J.R. Cordy, A.J. Malton and K.A. Schneider. Grammar Programming in TXL. IEEE 2nd International Workshop on Source Code Analysis and Manipulation (SCAM 2002), pp. 93-102, Oct. 2002.

[35] T.J. Biggerstaff. Design Recovery For Maintenance and Reuse, IEEE Computer, 22(7), pp. 36-99, July 1989. 